BMJ Open

Diabetes

Research

\& Care

\section{Type 2 diabetes exaggerates exercise effort and impairs exercise performance in older women}

To cite: Huebschmann AG, Kohrt WM, Herlache L, et al. Type 2 diabetes exaggerates exercise effort and impairs exercise performance in older women. BMJ Open Diabetes Research and Care 2015;3: e000124. doi:10.1136/ bmjdrc-2015-000124

Received 10 June 2015 Revised 19 August 2015 Accepted 9 September 2015

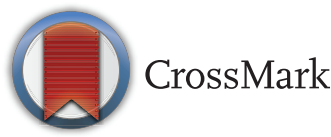

${ }^{1}$ Department of Medicine; Division of General Internal Medicine, University of Colorado (CU) School of Medicine (SOM), Aurora, Colorado, USA

${ }^{2}$ Center for Women's Health Research, Aurora, Colorado, USA

${ }^{3}$ Division of Geriatrics, Aurora, Colorado, USA ${ }^{4} \mathrm{CU}-\mathrm{SOM}$ Department of Biostatistics, Aurora, Colorado, USA

${ }^{5}$ Division of Cardiology, Aurora, Colorado, USA ${ }^{6}$ Division of Endocrinology, Aurora, Colorado, USA

Correspondence to Dr A G Huebschmann; amy.huebschmann@ ucdenver.edu

\section{ABSTRACT}

Objective: Type 2 diabetes mellitus (T2DM) is associated with high levels of disability and mortality. Regular exercise prevents premature disability and mortality, but people with T2DM are generally sedentary for reasons that are not fully established. We previously observed that premenopausal women with T2DM report greater effort during exercise than their counterparts without diabetes, as measured by the Rating of Perceived Exertion (RPE) scale. We hypothesized that RPE is greater in older women with T2DM versus no T2DM.

Research design and methods: We enrolled overweight, sedentary women aged $50-75$ years with $(\mathrm{n}=26)$ or without T2DM $(\mathrm{n}=28)$. Participants performed submaximal cycle ergometer exercise at $30 \mathrm{~W}$ and $35 \%$ of individually-measured peak oxygen consumption ( $35 \% \mathrm{VO}_{2}$ peak). We assessed exercise effort by RPE (self-report) and plasma lactate concentration.

Results: $\mathrm{VO}_{2}$ peak was lower in T2DM versus controls $(p=0.003)$. RPE was not significantly greater in T2DM versus controls ( $30 \mathrm{~W}$ : Control, 10.4 \pm 3.2 , T2DM, 11.7 $\pm 2.3, \mathrm{p}=0.08 ; 35 \% \mathrm{VO}_{2}$ peak: Control, $11.1 \pm 0.5$, T2DM, $12.1 \pm 0.5, p=0.21)$. However, lactate was greater in T2DM versus controls $(p=0.004$ at $30 \mathrm{~W} ; p<0.05$ at $35 \% \mathrm{VO}_{2}$ peak). Greater RPE was associated with higher lactate, higher heart rate, and a hypertension diagnosis ( $p<0.05$ at $30 \mathrm{~W}$ and $35 \% \mathrm{VO}_{2}$ peak).

Conclusions: Taken together, physiological measures of exercise effort were greater in older women with T2DM than controls. Exercise effort is a modifiable and thereby targetable end point. In order to facilitate regular exercise, methods to reduce exercise effort in T2DM should be sought.

Trial number: NCT00785005.

The prevalence of type 2 diabetes mellitus (T2DM) continues to rise worldwide and the highest prevalence rates are found among older adults. ${ }^{1}$ Exercise is considered a critical cornerstone of treatment for people with T2DM due to its beneficial effects on glycemic control, physical fitness, cardiovascular health and prevention of disability as well as premature mortality. ${ }^{2-4}$ However, people with T2DM are consistently more sedentary than

\section{Key messages}

- A novel barrier to physical activity that we have identified previously is that exercise feels more difficult to sedentary premenopausal women with type 2 diabetes mellitus (T2DM) than their similarly obese and sedentary counterparts without diabetes.

- We found statistically significant differences in plasma lactate during low- to moderate-intensity exercise in postmenopausal women with T2DM, as compared to their counterparts without diabetes; we also found clinically meaningful differences in exercise effort as measured subjectively by the Borg Rating of Perceived Exertion (RPE).

- An important take-home point for clinicians is to encourage patients to be physically active at a pace that is personally comfortable, as this tends to be associated with both good adherence and a good physiological fitness response.

similarly obese people who do not have diabetes, for reasons that are not clear. ${ }^{5}{ }^{6}$ Since lower fitness levels are linked to cardiovascular morbidity and mortality in populations with and without $\mathrm{T}_{2 \mathrm{DM}},^{7} 8$ understanding and overcoming barriers to physical activity for people with T2DM is critically important.

A novel barrier to physical activity that we have identified previously is that exercise feels more difficult to sedentary people with T2DM than their similarly obese and sedentary counterparts without diabetes. ${ }^{9} \quad 10$ Specifically, we have shown that effort during low intensity exercise is greater in premenopausal women with T2DM versus similarly obese controls, as measured by both the Borg Rating of Perceived Exertion (RPE) and plasma lactate concentrations during exercise. Since RPE is modifiable ${ }^{11-13}$ and plays a significant role in adherence to prescribed physical activity, ${ }^{14-16}$ it has great potential relevance as a modifiable barrier to physical activity. In addition, these findings may partly explain some barriers to physical 
activity identified in prior questionnaires and focus groups of people with T2DM, such as 'difficulty keeping up with others who don't have T2DM. ${ }^{17}$ Finally, RPE is related to the affective response to exercise, ${ }^{18} 19$ and the 2010 American Diabetes Association physical activity guidelines suggest that "affective responses to exercise may be important predictors of physical activity adoption and maintenance." ${ }^{3}$

Important possible mediators of increased exercise effort include impaired cardiorespiratory fitness levels and submaximal exercise responses. Submaximal exercise impairments include a slowed $\mathrm{VO}_{2}$ uptake kinetics response that represents a delay in achieving steady-state oxygen utilization during constant work rate exercise. Among adolescents and middle-aged adults, participants with T2DM have significantly worse peak cardiorespiratory fitness levels and worse submaximal exercise performance than their counterparts without diabetes. ${ }^{10} 20-22$ Less is known about these measures of exercise performance in older adults with $\mathrm{T}_{2} \mathrm{DM}^{23}$ even though older adults have the highest prevalence of diabetes. ${ }^{1}$ Therefore, we sought to compare measures of exercise effort and exercise performance during exercise in older women with T2DM versus their counterparts without diabetes. We hypothesized that there would be greater perceived effort during low to moderate intensity exercise in participants with T2DM compared to controls without diabetes. We also hypothesized that both fitness levels and submaximal exercise responses would be more impaired in participants with T2DM than controls, despite recruiting participants of similar weight and similar levels of habitual physical activity. We studied women with T2DM because T2DM confers greater exercise impairment in women than men. ${ }^{24}$

\section{RESEARCH DESIGN AND METHODS}

In this cross-sectional study, we enrolled overweight, sedentary women with or without T2DM from the metropolitan Denver, Colorado, USA community between 2007 and 2011. Participants were 50-75 years old with either T2DM $(n=26)$ or controls without diabetes $(n=28)$. We defined people without diabetes by the prevailing American Diabetes Association guidelines at the time of the research (ie, normal fasting glucose $<100 \mathrm{mg} / \mathrm{dL}$ and glycated hemoglibin, HbAlc $<6 \%) .{ }^{25}$ In addition, to minimize finding insulin resistance in control participants, we excluded controls with $>1$ first-degree relative with T2DM. ${ }^{25}$ Additional inclusion criteria ensured similar overweight and sedentary status in study groups: overweight/obese body mass index (BMI) $\left(25-39.9 \mathrm{~kg} / \mathrm{m}^{2}\right)$ and self-report of leisure physical activity behavior of $<60 \mathrm{~min} /$ week. Participants were postmenopausal as documented by no menses in $>12$ months and by measured follicle-stimulating hormone levels. ${ }^{10}$ Our exclusion criteria included conditions that imposed safety concerns or could impair exercise performance, such as uncontrolled hypertension, a history of atherosclerosis, congestive heart failure, autonomic or peripheral neuropathy, chronic disease of the lung, liver or kidneys, microalbuminuria, or tobacco use within 1 year. ${ }^{26}$ In addition, we excluded participants with prolonged duration of T2DM or suboptimal disease control: HbAlc $>8.5 \%$, duration of diabetes $>20$ years, microalbuminuria (urine microalbumin/creatinine $>30) .{ }^{27}$ Use of insulin, thiazolidinediones, GLP-1 agonists and DPP-4 inhibitors was excluded since these drugs might either affect exercise capacity or suggest more advanced disease. ${ }^{22} 28$

\section{Exercise effort}

Measures of exercise effort included RPE (primary outcome) and plasma lactate concentration (secondary outcome) measured during eight bouts of constant work rate submaximal exercise. The Borg RPE scale is the gold standard measure of RPE during exercise in healthy $^{29} 30$ and diabetic populations. ${ }^{31}$ The range of scores on this ordinal scale is from 6 to 20 with verbal anchors every two points (eg, RPE $=11$, 'light', RPE $=13$, 'somewhat hard'). To minimize bias, participants and the research staff recording RPE were blinded to the work rate. Plasma lactate concentration was measured using the lactate dehydrogenase method on blood drawn in perchloric acid tubes. ${ }^{10}$ RPE and lactate concentration were measured on two separate study dates at both an absolute work rate ( $30 \mathrm{~W}, 4$ bouts) and a relative work rate (4 bouts). We designed the relative work rate to be $35 \%$ of the $\mathrm{VO}_{2}$ peak from each participant's peak exercise test, in order to account for the influence of cardiorespiratory fitness on exercise effort. We chose $35 \% \mathrm{VO}_{2}$ peak as the relative work rate to ensure participants achieved a steady-state $\mathrm{VO}_{2}$ level that was reliably below yet close to the lactate threshold. In our prior published work in premenopausal women, $35 \%$ $\mathrm{VO}_{2}$ peak was $\sim 30 \mathrm{~W}$ for participants with T2DM. ${ }^{9}$

\section{Nutritional and body composition assessments prior to exercise testing}

To control for effects of diet on exercise performance, participants consumed a eucaloric study diet with standardized macronutrient distributions for 3 days prior to exercise testing and fasted for at least $4 \mathrm{~h}$ prior to exercise testing. ${ }^{10}$ Eucaloric diets were developed by registered dieticians based on body composition assessed by Dual-energy X-ray Absorptiometry (DXA scan, Hologic/ Discovery W, Hologic Inc, Bedford, Massachusetts, USA). DXA was also used to assess the exercise effort-related covariate of total fat-free mass.

\section{Exercise testing}

The exercise testing procedures have been described in detail previously. ${ }^{10}{ }_{32}$ In brief, each exercise test began with the participant seated upright at rest for $3 \mathrm{~min}$ on the cycle ergometer (Lode ergometer, MedGraphics, Minneapolis, Minnesota, USA) breathing into a mouthpiece connected to a metabolic cart (Ultima CPX, MedGraphics). Participants first performed a 
familiarization graded cycle ergometer exercise test (GXT). On a subsequent day, we used a ramping protocol GXT to measure $\mathrm{VO}_{2}$ peak. During the ramping protocol GXT, the rate of increase of the work rate to peak exercise capacity was individualized to ensure an optimal test duration of 10-14 min (eg, if the familiarization GXT peak work rate was $55 \mathrm{~W}$, the wattage increased continuously by $0.0833 \mathrm{~W} / \mathrm{s}$ to reach a work rate of $55 \mathrm{~W}$ at $11 \mathrm{~min}$ ). During exercise, $\mathrm{VO}_{2}$ was measured breath-by-breath and time-averaged over $30 \mathrm{~s}$ intervals. We defined $\mathrm{VO}_{2}$ peak by standard convention as the peak $\mathrm{VO}_{2}$ associated with an RER $\geq 1.1$ or as a $\mathrm{VO}_{2}$ plateau despite an increase in workload. ${ }^{33}$

\section{Exercise performance measures and other predictors of exercise effort}

Our secondary outcomes of exercise performance included cardiorespiratory fitness $\left(\mathrm{VO}_{2}\right.$ peak $)$ and a measure of submaximal exercise response (time constant $\left(\tau_{2}\right)$ from $\mathrm{VO}_{2}$ uptake kinetics) at both $30 \mathrm{~W}$ and $35 \% \mathrm{VO}_{2}$ peak.

\section{Kinetics measurements during constant-load exercise}

On subsequent study dates, participants performed eight bouts of submaximal constant-load exercise with work rates alternating between $30 \mathrm{~W}$ and $35 \% \mathrm{VO}_{2}$ peak. Each exercise bout was $8 \mathrm{~min}$ in duration. The research assistants recording the data and participants were blinded to the work rate. During constant-load exercise, we assessed submaximal exercise response using $\mathrm{VO}_{2}$ kinetics, where a longer time constant $\left(\tau_{2}\right)$ represents a longer time to achieve steady-state $\mathrm{VO}_{2} \cdot{ }^{10}$ As previously described, for each work rate, gas-exchange data for kinetic analyses were processed using a software program developed in our laboratory. ${ }^{10} 32$ The pulmonary $\mathrm{VO}_{2}$ kinetic responses data for each of the bouts of oxygen consumption were evaluated using a twocomponent exponential model. ${ }^{26}$

\section{Physiological predictors of exercise effort}

According to our conceptual model (figure 1), we considered the following physiological variables as potential predictors of exercise effort: heart rate during exercise, $\mathrm{VO}_{2}$ peak and $\tau_{2}$. In addition, we assessed metabolic and vascular factors that we hypothesized were likely to impair the physiological response to exercise in people with T2DM: Homeostasis Model Assessment of Insulin Resistance (HOMA-IR), ${ }^{34}$ markers of endothelial dysfunction such as a diagnosis of hypertension and arterial stiffness expressed as pulse-wave velocity ${ }^{35}$ (SphygmaCor CP system, AtCor Medical). HOMA-IR was calculated per standard convention from blood glucose and insulin measurements collected during a $12 \mathrm{~h}$ fast. ${ }^{34}$ We also measured glucose levels during the $8 \mathrm{~min}$ of exercise as an additional potential predictor of effort. A diagnosis of hypertension was obtained by self-report and validated during the medical history and physical examination by the study physician/PI (AGH). Pulse-wave velocity was assessed after a $4 \mathrm{~h}$ fast and prior to any exercise testing performed in the same study visit.

\section{Behavioral predictors of exercise effort}

According to our conceptual model where behavioral factors may influence the interpretation of physiological cues (figure 1), we also considered the following behavioral variables as potential predictors of RPE: self-efficacy to perform physical activity ${ }^{36-38}$ and depressive symptoms. ${ }^{39}$ We assessed these behavioral variables by paper surveys conducted prior to any exercise testing performed in the same study visit.

\section{Power calculation}

Based on our preliminary data where we observed an $\mathrm{RPE}$ difference of $1.3 \pm 1.4 \quad($ mean $\pm \mathrm{SD})$ between the T2DM and overweight control groups, ${ }^{9}$ we estimated that 54 participants would provide $82 \%$ power to detect a between-group difference in RPE of 0.8 in the $8 \mathrm{~min}$ of exercise at the 0.05 level. We considered a between-group RPE difference of one point to be clinically meaningful. ${ }^{41}$

\section{Statistical analysis}

All outcome and predictor variables were examined with descriptive statistics and graphic summaries, overall and by T2DM status. To determine group differences in RPE and lactate, $30 \mathrm{~W}$ data collected during four separate bouts of exercise and $35 \% \mathrm{VO}_{2}$ peak data collected during four separate bouts of exercise were evaluated using separate maximum likelihood repeated measures models (eg, $30 \mathrm{~W}$ model assessed 4 repeated measures). ${ }^{42}$ The primary outcomes were RPE and lactate at minute 8 . We also assessed changes in RPE within and between-groups across minutes 2, 4, 6 and 8 at $30 \mathrm{~W}$ and $35 \% \mathrm{VO}_{2}$ peak to determine if RPE levels were at steady-state, defined as equivalent levels at minutes 6 and 8 , and to compare the RPE change from minutes 2 to 8 by study group. We also assessed lactate concentrations at rest prior to exercise and the change in lactate concentrations from the resting baseline to the $8 \mathrm{~min}$ of exercise ( $\Delta$ lactate), by using separate maximum likelihood repeated measures models. Finally, we compared group differences in heart rate, also using a maximum likelihood repeated measures model at each of 2, 4, 6 and $8 \mathrm{~min}$ into the exercise bouts. To ensure that our data at the relative work rate of $35 \% \mathrm{VO}_{2}$ peak was accounting for fitness levels appropriately, we also conducted a sensitivity analysis to estimate group differences in effort variables (ie, RPE, lactate during minute 8 , heart rate during minute 8 of exercise) with adjustment for fitness at the $30 \mathrm{~W}$ absolute work rate for comparison with the group differences from the relative work rate of $35 \% \mathrm{VO}_{2}$ peak that accounted for fitness. We also conducted a sensitivity analysis to estimate group differences in resting lactate concentration with adjustment for fitness. 
Figure 1 Conceptual model of perceived effort during exercise.

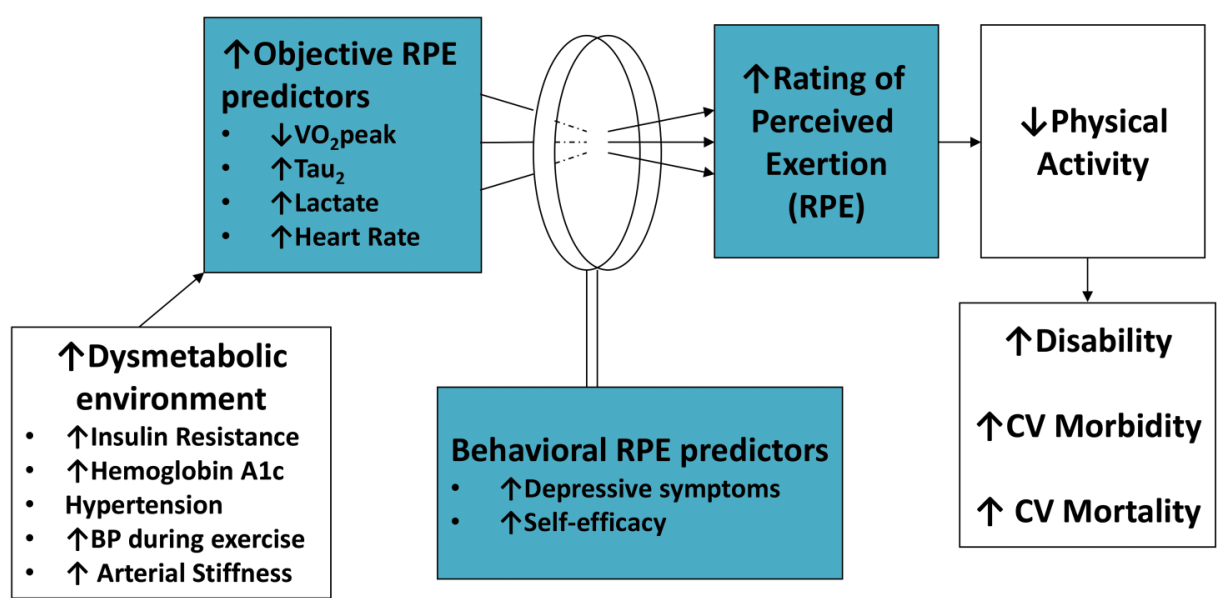

(figure 2A, B). Of note, despite all participants reaching steady-state oxygen uptake levels within the first $2 \mathrm{~min}$ of exercise, the RPE levels continued to rise during $8 \mathrm{~min}$ of exercise and had not yet reached steady-state at $8 \mathrm{~min}$ of exercise (the RPE slope was different from zero $(\mathrm{p}<0.001)$ from minutes 6 to 8 in each study group). The change in RPE from minute 2 to 8 was greater, but not significantly so, in the T2DM group compared to the control group $(\mathrm{p}=0.07)$.

Lactate concentrations are shown in figure $2 \mathrm{C}$. To summarize, mean lactate levels during minute 8 of exercise were significantly greater in the T2DM group as compared with control women ( $\mathrm{p}=0.004$ at $30 \mathrm{~W}$ and $\mathrm{p}=0.046$ at $35 \%$ $\mathrm{VO}_{2}$ peak, figure 2C). Mean lactate levels were also significantly greater at rest in the T2DM group compared with control women $(p<0.0001)$. The difference between resting and exercise lactate concentrations ( $\Delta$ lactate) was not significantly greater in the T2DM group compared with control women $(30 \mathrm{~W} \Delta$ lactate: $0.53 \pm 0.08$ vs $0.37 \pm 0.08$ $(\mathrm{p}=0.17) ; 35 \% \mathrm{VO}_{2}$ peak $\Delta$ lactate: $0.62 \pm 0.08$ vs $0.60 \pm 0.08$, $\mathrm{p}=0.86$ ). Our sensitivity analysis showed that group differences in resting lactate remained significantly different after adjustment for $\mathrm{VO}_{2}$ peak $(\mathrm{p}<0.001)$, and we observed no statistical association between $\mathrm{VO}_{2}$ peak and resting lactate concentration $(\mathrm{p}=0.98)$.

\section{Physiological effort-related variables}

$\mathrm{VO}_{2}$ peak was significantly lower in the T2DM group versus study group without diabetes by absolute and weightadjusted measures (table 1 ). However, $\tau_{2}$ was not different between study groups at $30 \mathrm{~W}$ or $35 \% \mathrm{VO}_{2}$ peak (table 1). Of the physiological variables that were prespecified as candidate predictors of RPE (figure 1), those that were significantly different between study groups included heart rate during exercise, lactate levels during exercise, $\mathrm{VO}_{2}$ peak, HbA1c and HOMA-IR (table 1). In a sensitivity analysis adjusting for fitness levels as $\mathrm{VO}_{2}$ peak $(\mathrm{mL} / \mathrm{min})$ rather than accounting for fitness differences at the relative work rate of $35 \% \mathrm{VO}_{2}$ peak, mean lactate levels and heart rate during exercise at $30 \mathrm{~W}$ remained significantly greater in the T2DM group as compared with control women ( $\mathrm{p}=0.02$ for lactate; $\mathrm{p}=0.01$ for heart rate). 


\begin{tabular}{|c|c|c|c|}
\hline Variable & $\begin{array}{l}\text { Overweight control } \\
(\mathrm{n}=28)\end{array}$ & $\begin{array}{l}\text { Type } 2 \text { diabetes (T2DM) } \\
(n=26)\end{array}$ & p Value \\
\hline Duration of T2DM, years (SD) & NA & $5.1(5.0)$ & NA \\
\hline Age, years (SD) & $59.8(5.8)$ & $59.3(5.7)$ & 0.39 \\
\hline Ethnicity, n (\% Hispanic) & $1(4)$ & $3(12)$ & 0.34 \\
\hline Race, n (\% Caucasian) & $27(96)$ & $18(67)$ & 0.01 \\
\hline \multicolumn{4}{|l|}{ Hypothesized physiological RPE predictors } \\
\hline $\mathrm{BMI}, \mathrm{kg} / \mathrm{m}^{2}$ (SD) & $30.1(2.8)$ & $31.3(3.9)$ & 0.25 \\
\hline $\mathrm{HbA} 1 \mathrm{c}, \%$ & $5.7(0.3)$ & $6.8(0.6)$ & $<0.001$ \\
\hline $\begin{array}{l}\text { Homeostasis Model Assessment of Insulin Resistance } \\
\text { (HOMA-IR) }\end{array}$ & $4.0(1.9)$ & $6.7(4.6)$ & 0.01 \\
\hline HTN prevalence (\%) & 46 & 50 & 0.99 \\
\hline Pulse wave velocity (m/s) & $9.2(0.7)$ & $9.9(0.8)$ & 0.06 \\
\hline Reported usual physical activity, MET h/week (SD) & $222(35)$ & $231(25)$ & 0.32 \\
\hline $\mathrm{VO}_{2}$ peak $(\mathrm{mL} / \mathrm{min})$ & $1470(286)$ & $1313(244)$ & 0.02 \\
\hline $\mathrm{VO}_{2}$ peak $(\mathrm{mL} / \mathrm{kg} / \mathrm{min})$ & $17.8(3.0)$ & $15.4(2.5)$ & 0.003 \\
\hline Work rate during $35 \% \mathrm{VO}_{2}$ peak (SD) & $43.5(7.7)$ & $35.9(7.1)$ & $<0.0001$ \\
\hline Heart rate during $8 \mathrm{~min}$ of exercise at $30 \mathrm{~W}(\mathrm{bpm})$ & $91(2)$ & $102(2)$ & 0.002 \\
\hline Heart rate during $8 \mathrm{~min}$ of exercise at $35 \% \mathrm{VO}_{2}$ peak & $98(3)$ & $105(3)$ & 0.04 \\
\hline Resting lactate concentrations (mmol/L) & $0.39(0.03)$ & $0.65(0.03)$ & $<0.0001$ \\
\hline Lactate during $8 \mathrm{~min}$ of exercise at $30 \mathrm{~W}(\mathrm{mmol} / \mathrm{L})$ & $0.76(0.10)$ & $1.18(0.10)$ & 0.004 \\
\hline Lactate at $8 \mathrm{~min}$ at $35 \% \mathrm{VO}_{2}$ peak (mmol/L) & $0.99(0.10)$ & $1.28(0.10)$ & 0.047 \\
\hline$\tau_{2}$ at $30 \mathrm{~W}$ & $39.2(13)$ & $36.2(9.1)$ & 0.34 \\
\hline$\tau_{2}$ at $35 \% \mathrm{VO}_{2}$ peak & $38.6(12.2)$ & $38.5(10.2)$ & 0.98 \\
\hline \multicolumn{4}{|l|}{ Hypothesized behavioral RPE predictors } \\
\hline \multicolumn{4}{|l|}{ Depressive symptoms } \\
\hline CES-D & $11.4(3.7)$ & $11.7(5.6)$ & 0.77 \\
\hline PHQ-9 & $3.3(3.3)$ & $3.2(3.2)$ & 0.96 \\
\hline \multicolumn{4}{|l|}{ Self-efficacy scores } \\
\hline Endurance self-efficacy & $746(359)$ & $739(264)$ & 0.94 \\
\hline Lorig self-efficacy & $481(95)$ & $479(94)$ & 0.92 \\
\hline Sallis self-efficacy & $45(8)$ & $47(8)$ & 0.23 \\
\hline
\end{tabular}

Data expressed as mean (SE) for heart rate, lactate; data expressed as mean (SD) for all other continuous variables.

Missing data for $<10 \%$ of participants with the exception of pulse wave velocity where we are missing $40 \%$ of data for each study group. BMI, body mass index; CES-D, Center for Epidemiological Studies Depression; HbA1c, glycated hemoglobin; HTN, hypertension; PHQ-9, Patient Health Questionnaire-9; RER, respiratory exchange ratio; T2DM, type 2 diabetes mellitus.

\section{Association of physiological variables with effort}

In the regression model assessing predictors of RPE in the 8th min of exercise with adjustment for insulin resistance by HOMA-IR, the physiological variables significantly associated with RPE were heart rate during exercise, diagnosis of hypertension and plasma lactate during exercise (table 2). Although HOMA-IR was not a statistically significant predictor of RPE, it was included in the model as a precision efficiency variable (ie, the model fit was enhanced by including HOMA-IR, and HOMA-IR outperformed the other metabolic candidate variables of T2DM diagnosis, glucose level during exercise and $\mathrm{HbAlc}$ ). In the regression model assessing predictors of lactate level at minute 8 , the only significant predictor at both $30 \mathrm{~W}$ and $35 \%$ VO2peak was heart rate during exercise. At the $30 \mathrm{~W}$ work rate only, $\tau_{2}$ was also significantly associated with lactate.

\section{Behavioral effort-related variables}

We found no significant difference in depressive symptoms or self-efficacy to perform physical activity by study group (table 1). In a multivariate regression model assessing predictors of RPE with adjustment for HOMA-IR, we found that depressive symptoms as measured by the Center for Epidemiological Studies-Depression (CESD) ${ }^{39}$ scale were associated with RPE at $30 \mathrm{~W}$ but not at $35 \% \quad \mathrm{VO}_{2}$ peak. Depressive symptoms as measured by the PHQ-9 $9^{40}$ scale were not predictive of RPE at either work rate.

\section{DISCUSSION}

We found that objective measures of exercise effort during submaximal exercise of lactate and heart rate during exercise were significantly greater in older women with T2DM compared to controls without diabetes. In addition, we identified higher levels of perceived effort in women with T2DM than in controls without diabetes, but these group differences in RPE were not statistically significant. Although RPE did not differ significantly between study groups, the RPE group differences observed at both $30 \mathrm{~W}$ and $35 \% \quad \mathrm{VO}_{2}$ peak were $\geq 1$ point-others have reported a 1-point 

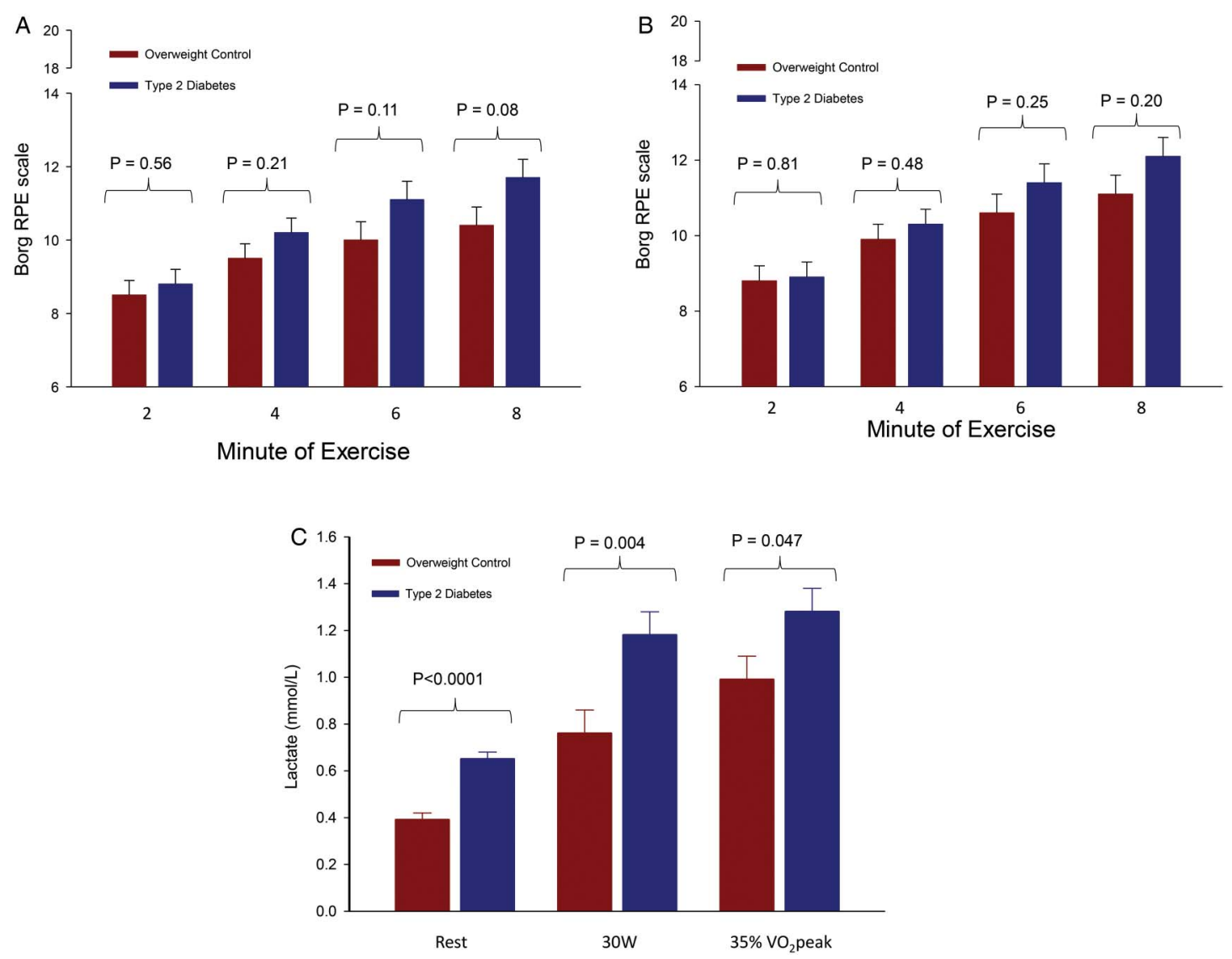

Figure 2 (A) Group differences in rating of perceived exertion over time at $30 \mathrm{~W}$. (B) Group differences in rating of perceived exertion over time at $35 \% \mathrm{VO}_{2}$ peak. (C) Higher lactate concentrations in T2DM during rest and exercise.

difference to be clinically meaningful on the RPE scale. ${ }^{44}$ In addition, we found both heart rate and lactate during exercise to be significant predictors of RPE in the multivariate analysis, supporting the existing research that these three measures are all related markers of effort. ${ }^{30} 45$ Taken together, these measures suggest that women with T2DM experience exercise, even at relatively low levels, to be more strenuous than their counterparts without diabetes. Exercise effort is an important barrier to physical activity because it is modifiable ${ }^{11-13}$ and the perception of more intense effort during exercise has been associated with lower levels of usual physical activity. ${ }^{14-16}$

As has been previously shown in adolescents and adults with T2DM, we found that mean levels of peak cardiorespiratory fitness were significantly lower in women with T2DM than in controls. ${ }^{10} 202223$ Similar to the finding of Wilkerson et $a l^{23}$ in older men, but different from our findings in younger adults, ${ }^{10} 22$ the present study found no differences in mean $\tau_{2}$ levels in the T2DM group as compared to controls (table 1). Of note, the values of $\tau_{2}$ in our control participants were longer than those observed in prior studies of adolescent and premenopausal control participants, ${ }^{22} 2432$ suggesting that the kinetic differences observed in younger T2DM may make them physiologically 'older' compared to their similarly-aged peers. Conversely, the lack of difference in subjects with T2DM versus controls in older populations (eg, Wilkerson $e t a l^{23}$ and the present study) suggests that age-related changes in this response parameter may mask the independent effects of T2DM.

Few studies have compared RPE in people with T2DM to RPE in people without diabetes. The existing literature suggests that RPE differences by T2DM status are more likely to be apparent at absolute work rates than at relative work rates that account for the lower fitness levels exhibited in people with T2DM than controls. ${ }^{9} 46$ In our prior study of premenopausal women, we assessed RPE at two absolute work rates of $20 \mathrm{~W}$ and $30 \mathrm{~W}$ during cycle ergometer exercise and found significantly greater RPE in women with T2DM when compared to both overweight controls and normal weight controls with no T2DM. ${ }^{9}$ Coquart et $a l^{46}$ studied overweight women with T2DM and overweight controls at a relative work rate of $100 \%$ ventilatory threshold (mean work rate in watts: T2DM: 41 \pm 14 ; overweight control: $43 \pm 11)$ and found no significant group differences in RPE (T2DM=13.7 \pm 2.3 , overweight control=13.2 \pm 1.6$)$. Consistent with the concept that RPE differences are more attenuated at relative work rates than absolute work rates, in the present study the RPE differences between T2DM women and controls were smaller at the 
Table 2 Regression models for predictors of RPE and lactate at $30 \mathrm{~W}$ and at $35 \% \mathrm{VO}_{2}$ peak

\begin{tabular}{|c|c|c|}
\hline Effort predictor variables & 30 W F statistic (p value) & $35 \% \mathrm{VO}_{2}$ peak $\mathrm{F}$ statistic ( $\mathrm{p}$ value) \\
\hline \multicolumn{3}{|l|}{ Physiological RPE predictor model } \\
\hline Heart rate during 8 min exercise & $9.8(p=0.002)$ & $4.1(p=0.045)$ \\
\hline Hypertension diagnosis (yes/no) & $6.4(p=0.02)$ & $10.3(p=0.003)$ \\
\hline Lactate during $8 \mathrm{~min}$ exercise & $6.0(p=0.02)$ & $4.2(p=0.04)$ \\
\hline HOMA-IR & $2.3(p=0.14)$ & $0.8(p=0.38)$ \\
\hline \multicolumn{3}{|l|}{ Behavioral RPE predictor model } \\
\hline CES-D & $4.5(p=0.04)$ & $3.2(p=0.08)$ \\
\hline HOMA-IR & $2.5(p=0.11)$ & $1.5(p=0.23)$ \\
\hline \multicolumn{3}{|l|}{ Physiological lactate predictor model } \\
\hline Heart rate during 8 min exercise & $5.6(p=0.02)$ & $8.9(p=0.0002)$ \\
\hline T2DM diagnosis (yes/no) & $6.2(p=0.02)$ & $1.6(p=0.26)$ \\
\hline
\end{tabular}

relative work rate of $35 \% \quad \mathrm{VO}_{2}$ peak than at $30 \mathrm{~W}$, although the group differences were not statistically significant at either $30 \mathrm{~W}(\mathrm{p}=0.08)$ or at $35 \% \mathrm{VO}_{2}$ peak $(\mathrm{p}=0.20)$.

After adjustment for insulin resistance in our population of overweight women with and without T2DM, we found that the objective markers of effort of plasma lactate and heart rate during exercise were very strong predictors of RPE, as has been observed in other healthy populations. ${ }^{47}$ A somewhat surprising finding was that RPE was more strongly associated with a diagnosis of hypertension than with heart rate and plasma lactate during exercise. This finding warrants confirmation in future studies to ensure that it is not spurious. Others have reported an association between glucose levels and RPE during high-intensity exercise of prolonged duration because the 'depletion of carbohydrate fuel sources triggers muscular fatigue'. ${ }^{47}$ We did not find an association between glucose levels during exercise and RPE, thus supporting the existing literature that energy substrates such as glucose do not appear to influence RPE at lower exercise intensities. ${ }^{47}$

Our findings add to the literature suggesting that lactate levels during rest and exercise are greater in people with T2DM than in their counterparts without diabetes. Previous studies of differences in lactate levels during exercise in people with T2DM versus controls have been conducted in younger women ${ }^{10}$ and middle-aged men, ${ }^{48}$ but we are not aware of other studies in older women with T2DM versus no diabetes. Resting lactate concentrations were greater in people with T2DM as compared to controls without diabetes in prior studies as well as the present study-elevated lactate at rest has even been recognized as a predictor of incident T2DM. ${ }^{49} 50$ In prior studies by Regensteiner and Mogensen et al, the lactate concentrations during exercise were significantly greater in the T2DM group versus overweight control group, and the differences became larger at higher work rates. With moderate intensity exercise, lactate levels may modestly increase as we observed, and lactate dehydrogenase (LDH) activity increases and can offset/counter-balance hydrogen ion production in order to delay muscle fatigue. ${ }^{51}$ Our observations of higher absolute lactate levels at rest and during exercise may indicate an alteration of the $\mathrm{LDH}$ complex and function in T2DM-or perhaps a different position on the $\mathrm{LDH}$ complex operating curve. We observed wide interindividual variance in $\Delta$ lactate concentrations and were not powered to detect significance in these data. The wide interindividual variance in our observed $\Delta$ lactate concentrations may relate partly to the effect of different $\mathrm{LDH}$ isoforms and partly to variance in exercise intensity perturbation. In other studies assessing metabolic predictors of exercise effort, absolute lactate concentrations during exercise have been used as RPE predictors rather than $\Delta$ lactate concentrations. ${ }^{30} 45$ A scientific rationale for using the absolute concentrations of lactate rather than $\Delta$ lactate is that lactate is dynamically produced and metabolized at rest and during exercise and levels of metabolism are proportionally higher at greater lactate concentrations. ${ }^{52-54}$ Thus, the absolute lactate concentration provides a meaningful comparison of the absolute difference between lactate production and metabolism at any given time. The 2010 American Diabetes Association physical activity guidelines suggest that "affective responses to exercise may be important predictors of physical activity adoption and maintenance". 3 Perceptions of disproportionately greater effort during exercise may be one reason that people with T2DM are less active than their counterparts without T2DM, as perceptions of exercise effort over a certain threshold worsen the affective response to exercise and are linked to lower levels of leisure physical activity. ${ }^{14-16}$ Our effort measures were obtained at work rates that are less intense than many activities of daily living. Since people tend to prefer physical activities with an intensity in the $11-14$ range, ${ }^{55}$ our findings provide some additional support to existing concerns 
that overweight, sedentary individuals with and without T2DM may avoid activities of daily living because they are perceptually too difficult and hence unpleasant. For example, our study population of overweight, sedentary women that reported mean RPE levels in the 10-12 range during cycle ergometry at $30 \mathrm{~W}$ (approximately 3 METs of intensity) would be expected to experience a much higher RPE while walking up a flight of stairs that represents approximately 10 METs of intensity. ${ }^{57}$ Thus, activities of daily living that require greater intensity activity, such as walking up stairs or walking at a faster pace to keep up with others, may be avoided for individuals such as those we studied. In keeping with this theory, a prior barrier reported by people with T2DM is the inability to keep pace with others who do not have diabetes. ${ }^{17}$ A recent review article suggested that practitioners balance goals for their patients to conduct regular exercise in a range of intensity that will allow for physiological improvement and the need to make physical activity 'palatable' in an intensity range that is acceptable to the individual. ${ }^{58}$ An important take-home point for clinicians is to encourage patients to be physically active at a pace that is personally comfortable as this tends to be associated with both good adherence and a good physiological fitness response. ${ }^{58} 59$

The strengths of this study include the assessment of effort during exercise by both subjective (RPE) and objective methods (plasma lactate and heart rate), as well as assessments of both physiological and behavioral predictors of exercise effort. The cross-sectional nature of the study prevented us from drawing any causal inferences for the statistical associations that we observed between exercise effort and the predictors of effort. Although the control subjects did not meet a diagnosis of prediabetes by the prevailing criteria of the time, a subset would have met the current criteria for prediabetes by a HbA1c of 5.7-6.4\%. The inclusion of control subjects with prediabetes may have diminished the differences in exercise effort observed between the study groups. Another limitation of these findings is that the duration of exercise bouts assessed was only $8 \mathrm{~min}$; evaluating the effort response to longer bouts of duration would also be important to examine in future studies.

In summary, we found what others have reported to be clinically meaningful differences in $\mathrm{RPE}^{44}$ and statistically significant differences in lactate and heart rate during low-to moderate-intensity exercise in postmenopausal women with T2DM, as compared to their counterparts without diabetes. The group differences in RPE were not as large as we observed in our prior study of younger women, ${ }^{9}$ suggesting that future studies in older adults should use a more conservative estimate of effect size than is appropriate in younger women. It is possible that the effects of aging may influence T2DM-related exercise impairments. Greater perceived exertion is a modifiable and thereby targetable end point. Therefore, methods to reduce perception of work effort in T2DM should be sought in order to facilitate regular physical activity in people with T2DM. Improving physical activity would also improve the premature disability and mortality experienced by people with T2DM.

Acknowledgements This work was supported by funding from the University of Colorado Center for Women's Health Research, the University of Colorado Division of General Internal Medicine and the Eugene $\mathrm{C}$ and Florence Armstrong Family Foundation. During the period of this project, AGH was supported by NIH/NCATS Colorado CTSA Grant Number KL2 TR001080.

Contents are the authors' sole responsibility and do not necessarily represent official NIH views. The authors have no relevant conflicts of interest to disclose. The authors very much appreciate the participation of our volunteer study participants.

Contributors AGH and LH collected the data. AGH analyzed and interpreted the data and drafted the manuscript. PW supervised the statistical data analysis and revised the manuscript. JEBR, TAB, WMK, SD and JGR interpreted the data and revised the manuscript. All authors read and approved the final version of the manuscript. AGH is the guarantor of this work and, as such, had full access to all the data in the study and takes responsibility for the integrity of the data and the accuracy of the data analysis.

Funding University of Colorado School of Medicine; Eugene C and Florence Armstrong Family Foundation; National Institutes of Health.

Competing interests None declared.

Provenance and peer review Not commissioned; externally peer reviewed.

Data sharing statement No additional data are available.

Ethics statement The study consent processes and procedures were approved by the University of Colorado Institutional Review Board.

Open Access This is an Open Access article distributed in accordance with the terms of the Creative Commons Attribution (CC BY 4.0) license, which permits others to distribute, remix, adapt and build upon this work, for commercial use, provided the original work is properly cited. See: http:// creativecommons.org/licenses/by/4.0/

\section{REFERENCES}

1. Centers for Disease Control and Prevention. National diabetes fact sheet: general information and national estimates on diabetes in the United States, 2011. Atlanta, GA: U.S. Department of Health and Human Services, Centers for Disease Control and Prevention, 2012.

2. Wing RR, Bolin P, Brancati FL, et al., Look AHEAD Research Group. Cardiovascular effects of intensive lifestyle intervention in type 2 diabetes. N Engl J Med 2013;369:145-54.

3. Colberg SR, Albright AL, Blissmer BJ, et al. Exercise and type 2 diabetes: American College of Sports Medicine and the American Diabetes Association: joint position statement. Exercise and type 2 diabetes. Med Sci Sports Exerc 2010;42:2282-303.

4. Huffman KM, Sloane R, Peterson MJ, et al. The impact of self-reported arthritis and diabetes on response to a home-based physical activity counselling intervention. Scand J Rheumatol 2010;39:233-9.

5. Morrato EH, Hill JO, Wyatt HR, et al. Physical activity in US Adults with diabetes and at risk for developing diabetes, 2003. Diabetes Care 2007;30:203-9.

6. Zhao G, Ford ES, Li C, et al. Physical activity in US older adults with diabetes mellitus: prevalence and correlates of meeting physical activity recommendations. J Am Geriatr Soc 2011;59:132-7.

7. Blair SN, Kampert JB, Kohl HW III Jr, et al. Influences of cardiorespiratory fitness and other precursors on cardiovascular disease and all-cause mortality in men and women. JAMA 1996;276:205-10.

8. Church TS, Cheng YJ, Earnest CP, et al. Exercise capacity and body composition as predictors of mortality among men with diabetes. Diabetes Care 2004;27:83-8.

9. Huebschmann AG, Reis EN, Emsermann C, et al. Women with type 2 diabetes perceive harder effort during exercise than nondiabetic women. Appl Physiol Nutr Metab 2009;34:851-7.

10. Regensteiner JG, Bauer TA, Reusch JE, et al. Abnormal oxygen uptake kinetic responses in women with type II diabetes mellitus. J Appl Physiol 1998;85:310-17. 
11. Lacharité-Lemieux M, Brunelle JP, Dionne IJ. Adherence to exercise and affective responses: comparison between outdoor and indoor training. Menopause 2015;22:731-40.

12. Chan C, Driscoll T, Ackermann BJ. Effect of a musicians' exercise intervention on performance-related musculoskeletal disorders. Med Probl Perform Art 2014;29:181-8.

13. Skrinar GS, Ingram SP, Pandolf KB. Effect of endurance training on perceived exertion and stress hormones in women. Percept Mot Skills 1983;57(3 Pt 2):1239-50.

14. Dishman RK, Buckworth J. Increasing physical activity: a quantitative synthesis. Med Sci Sports Exerc 1996;28:706-19.

15. Sallis JF, Haskell WL, Fortmann SP, et al. Predictors of adoption and maintenance of physical activity in a community sample. Prev Med 1986;15:331-41.

16. Perri MG, Anton SD, Durning PE, et al. Adherence to exercise prescriptions: effects of prescribing moderate versus higher levels of intensity and frequency. Health Psychol 2002;21:452-8.

17. Korkiakangas EE, Alahuhta MA, Laitinen JH. Barriers to regular exercise among adults at high risk or diagnosed with type 2 diabetes: a systematic review. Health Promot Int 2009;24:416-27.

18. Parfitt G, Eston R. Changes in ratings of perceived exertion and psychological affect in the early stages of exercise. Percept Mot Skills 1995;80:259-66.

19. Acevedo EO, Kraemer RR, Haltom RW, et al. Percentual responses proximal to the onset of blood lactate accumulation. J Sports Med Phys Fitness 2003:43:267-73.

20. Regensteiner JG, Sippel J, McFarling ET, et al. Effects of non-insulin-dependent diabetes on oxygen consumption during treadmill exercise. Med Sci Sports Exerc 1995;27:875-81.

21. Baldi JC, Aoina JL, Oxenham HC, et al. Reduced exercise arteriovenous $\mathrm{O} 2$ difference in type 2 diabetes. J Appl Physiol 2003;94:1033-8.

22. Nadeau KJ, Zeitler PS, Bauer TA, et al. Insulin resistance in adolescents with type 2 diabetes is associated with impaired exercise capacity. J Clin Endocrinol Metab 2009;94:3687-95

23. Wilkerson DP, Poole DC, Jones AM, et al. Older type 2 diabetic males do not exhibit abnormal pulmonary oxygen uptake and muscle oxygen utilization dynamics during submaximal cycling exercise. $\mathrm{Am}$ J Physiol Regul Integr Comp Physiol 2011;300:R685-92.

24. Regensteiner JG, Bauer TA, Huebschmann AG, et al. Sex differences in the effects of type 2 diabetes on exercise performance. Med Sci Sports Exerc 2015;47:58-65.

25. American Diabetes Association. Standards of medical care in diabetes-2007. Diabetes Care 2007;30(Suppl 1):S4-S41.

26. Bauer TA, Reusch JE, Levi M, et al. Skeletal muscle deoxygenation after the onset of moderate exercise suggests slowed microvascular blood flow kinetics in type 2 diabetes. Diabetes Care 2007;30:2880-5.

27. Estacio RO, Regensteiner JG, Wolfel EE, et al. The association between diabetic complications and exercise capacity in NIDDM patients. Diabetes Care 1998:21:291-5.

28. Regensteiner JG, Bauer TA, Reusch JE. Rosiglitazone improves exercise capacity in individuals with type 2 diabetes. Diabetes Care 2005:28:2877-83.

29. Borg GA. Psychophysical bases of perceived exertion. Med Sci Sports Exerc 1982;14:377-81.

30. Chen MJ, Fan X, Moe ST. Criterion-related validity of the Borg ratings of perceived exertion scale in healthy individuals: a meta-analysis. J Sports Sci 2002;20:873-99.

31. Kunitomi M, Takahashi K, Wada J, et al. Re-evaluation of exercise prescription for Japanese type 2 diabetic patients by ventilatory threshold. Diabetes Res Clin Pract 2000;50:109-15.

32. Brandenburg SL, Reusch JE, Bauer TA, et al. Effects of exercise training on oxygen uptake kinetic responses in women with type 2 diabetes. Diabetes Care 1999;22:1640-6.

33. Weber CT, Janicki JS, McElroy PA. Cardiopulmonary exercise (CPX) testing. In: Weber CT, Janicki JS, eds. Cardiopulmonary exercise testing. Philadelphia: W.B. Saunders Company, 1986:151-67.

34. Matthews DR, Hosker JP, Rudenski AS, et al. Homeostasis model assessment: insulin resistance and beta-cell function from fasting plasma glucose and insulin concentrations in man. Diabetologia 1985;28:412-19.
35. Cecelja M, Chowienczyk P. Dissociation of aortic pulse wave velocity with risk factors for cardiovascular disease other than hypertension: a systematic review. Hypertension 2009;54:1328-36.

36. Hu L, McAuley E, Motl RW, et al. Influence of self-efficacy on the functional relationship between ratings of perceived exertion and exercise intensity. J Cardiopulm Rehabil Prev 2007;27:303-8.

37. Lorig K, Chastain RL, Ung E, et al. Development and evaluation of a scale to measure perceived self-efficacy in people with arthritis. Arthritis Rheum 1989;32:37-44.

38. Sallis JF, Pinski RB, Grossman RM, et al. The development of self-efficacy scales for health-related diet and exercise behaviors. Health Educ Res 1988;3:10.

39. Radloff LS. The CES-D scale: a self-report depression scale for use in the general population. Appl Psychol Meas 1977;1:385-401.

40. Kroenke K, Spitzer RL, Williams JB. The PHQ-9: validity of a brief depression severity measure. J Gen Intern Med 2001;16:606-13.

41. Noble BJ, Robertson RJ. The Borg Scale: development, administration, and experimental use. In: Mittelmeier K, ed. Perceived exertion. Champaign: Human Kinetics, 1996:59-89.

42. Verbeke G, Molenberghs G. Linear mixed models in practice: a SAS oriented approach. New York, NY: Springer, 1997.

43. Sas Institute I. Base SAS ${ }^{2} 9.2$ Procedures Guide. Cary, NC: Sas Institute I, 2009.

44. Ries AL. Minimally clinically important difference for the UCSD Shortness of Breath Questionnaire, Borg Scale, and Visual Analog Scale. COPD 2005:2:105-10.

45. Scherr J, Wolfarth B, Christle JW, et al. Associations between Borg's rating of perceived exertion and physiological measures of exercise intensity. Eur J Appl Physiol 2013;113:147-55.

46. Coquart JB, Lemaire $\mathrm{C}$, Dubart $\mathrm{AE}$, et al. Intermittent versus continuous exercise: effects of perceptually lower exercise in obese women. Med Sci Sports Exerc 2008;40:1546-53.

47. Noble BJ, Robertson RJ. Peripheral and nonspecific physiological mediators. In: Mittelmeier K, ed. Perceived exertion. Champaign, IL: Human Kinetics, 1996:125-56.

48. Mogensen M, Vind BF, Højlund $\mathrm{K}$, et al. Maximal lipid oxidation in patients with type 2 diabetes is normal and shows an adequate increase in response to aerobic training. Diabetes Obes Metab 2009;11:874-83.

49. Ohlson LO, Larsson B, Björntorp $\mathrm{P}$, et al. Risk factors for type 2 (non-insulin-dependent) diabetes mellitus. Thirteen and one-half years of follow-up of the participants in a study of Swedish men born in 1913. Diabetologia 1988;31:798-805.

50. Crawford SO, Hoogeveen RC, Brancati FL, et al. Association of blood lactate with type 2 diabetes: the Atherosclerosis Risk in Communities Carotid MRI Study. Int J Epidemiol 2010;39:1647-55.

51. Ament W, Verkerke GJ. Exercise and fatigue. Sports Med 2009;39:389-422.

52. Finsterer J. Biomarkers of peripheral muscle fatigue during exercise. BMC Musculoskelet Disord 2012;13:218.

53. Ahlborg G, Hagenfeldt L, Wahren J. Substrate utilization by the inactive leg during one-leg or arm exercise. J Appl Physiol 1975;39:718-23.

54. Ahlborg G, Felig P. Lactate and glucose exchange across the forearm, legs, and splanchnic bed during and after prolonged leg exercise. J Clin Invest 1982;69:45-54.

55. Dishman RK. Prescribing exercise intensity for healthy adults using perceived exertion. Med Sci Sports Exerc 1994;26:1087-94.

56. Glass SC, Chvala AM. Preferred exertion across three common modes of exercise training. J Strength Cond Res 2001;15:474-9.

57. Kozey SL, Lyden K, Howe CA, et al. Accelerometer output and MET values of common physical activities. Med Sci Sports Exerc 2010;42:1776-84

58. Ekkekakis P, Parfitt G, Petruzzello SJ. The pleasure and displeasure people feel when they exercise at different intensities: decennial update and progress towards a tripartite rationale for exercise intensity prescription. Sports Med 2011;41:641-71.

59. Jakicic JM, Jaramillo SA, Balasubramanyam A, et al. Effect of a lifestyle intervention on change in cardiorespiratory fitness in adults with type 2 diabetes: results from the Look AHEAD Study. Int $J$ Obes (Lond) 2009;33:305-16. 